Vol. 1 No. 1:2

\section{Averting MERS-Cov Emerging Threat and Epidemics: The Importance of Community Alertness and Preparedness Policies and Programs}

\section{Abstract}

Africa being part of the global village is challenged by unprecedented and persistent emerging infectious diseases threats and outbreaks. Recent memories of the Middle East Respiratory Syndrome Coronavirus (MERS-CoV) outbreaks in Saudi Arabia (2012) and South Korea (2015), West Africa Ebola viral disease outbreak and public health humanitarian crisis (2014) and China's SARS outbreaks. Various differences and similarities between these diseases have triggered serious concerns and magnified that similar outbreaks could occur anywhere in the world with lingering health complications, socio-political and economic crises. Yet, there is dearth of knowledge on MERS-CoV reservoirs, risk factors of emergence, infectivity and transmissibility in Africa and globally. This paper highlights forward approaches and key activities to the African Union, governments and institutions and all stakeholders including public-private partnership. These play paramount roles in providing information and support to address MERS-CoV and other emerging infectious diseases research gaps, (2) promoting community alertness and resilience in preparedness, (3) fostering effective and coordinated logistics in risk communication, planning and response in MERS-CoV public mobilization literacy and awareness, advocacy and mitigation. There is an urgent need to establish an integrated and strategic evidence-based local and nationwide surveillance and response capacity, (5) better understand the MERS-CoV animal reservoir(s) and human-human infectivity and transmissibility, and (6) implement informed community-based or national threat and epidemics actionable emergency insurance facility and compensation schemes. Conclusively, improved early warning, active and integrated epidemiological and genomic surveillance indicators data gathering to inform and guide effective and community resilience capability in consolidating and upholding knowledge gains from "One Health Surveillance Network" and best practices in achieving "One Health" and global health security.
Ernest Tambo $0^{1,2}$, Tamiru Oljira ${ }^{3}$, Olalubi A. Oluwasogo ${ }^{4}$, Emad I. M. Khater 5,6, Zhou Xiao-Nong ${ }^{7,8}$

1 Faculté des Sciences Biomédicales et Pharmaceutiques, Université des Montagnes, Bangangté, République du Cameroun

2 Sydney Brenner Institute for Molecular Bioscience, Faculty of Health Sciences, University of the Witwatersrand, Johannesburg, South Africa

3 Department of Biology, College of Natural and Computational Sciences, Haramaya University, Ethiopia

4 Department of Public Health, Kwara State University (KWASU), Malete, Kwara, Federal Republic of Nigeria

5 Dallah Establishment, Dengue fever control project, Jeddah, Saudi Arabia

6 Department of Entomology, Faculty of Science, Ain Shams University, Cairo, Egypt

7 National Institute for Parasitic Disease, Chinese Center for Disease Control and Prevention, Shanghai, PR China

8 WHO, Collaborating Center in Malaria, Schistosomiasis and Filariasis, Shanghai, PR China

Corresponding author: Ernest Tambo

झtambo0711@gmail.com

Sydney Brenner Institute for Molecular Bioscience, Faculty of Health Sciences, University of the Witwatersrand, Johannesburg, South Africa

Tel: +41 (0)91 8119176

\section{Introduction}

Since 2012, an outbreak of Middle Eastern Respiratory Syndrome Coronavirus (MERS-CoV) has been recorded in 26 countries worldwide with the vast majority of these cases reported in the Kingdom of Saudi Arabia. Recently, South Korea has reported the second highest number of infections after Saudi Arabia with a total of 126 MERS-CoV cases, where 11 deaths [1]. Overall, a total of 906 laboratory-confirmed cases of MERS-CoV have been reported worldwide, including 361 deaths as of 21 October 2014 in Saudi Arabia. An overall South Korea's new cases bring the total of MERS cases globally to 1,289 , based on World Health Organization (WHO) data, with at least 455 related fatalities and laboratory confirmed cases, with alarming rate of patient deterioration, growing concerns on health and economic impact of the disease [2,3]. With an incubation period of 2-6 days following exposure ranging from 2-14 days, the recent memory of West Africa Ebola outbreak public health humanitarian crisis, 
SARS and MERS outbreak in China and South Korea respectively has magnified concerns that a similar outbreak could occur with triggering serious lingering health complications, social political and economic crises [4,5]. Previous studies have found that the MERS-CoV is responsible of up to $37-40$ percent of people infected; however, the number of people infected with the virus may have been underestimated because some people did not seek health care $[1,2,3,5]$. Vital approaches and response activities imperative to avert MERS-CoV and other emerging zoonotic diseases threat and epidemic in Africa and low and middle income countries (LMIC) is summarized [Table 1].

Hitherto, little is documented understood on MERS reservoir(s), risk factors of emergence, infectivity and transmissibility in Africa and globally in assessing MERS outbreak threats and outbreak severity in low and middle income countries (LMIC), especially Sub-Saharan Africa already challenged with the major killers (HIV) AIDS, TB and Malaria) and rising burden of non-communicable diseases (NCDs) [5,6] The increasing use of surveillance and monitoring systems as critical cornerstone in improving preventive and control measures and timely emergency response in epidemics has been advocated to support evidence-based risk / threat mitigation such as information and data gathering, social mobilization and community engagement, health education, awareness campaigns and technical support assistance $[7,8]$. Understanding the fundamental epidemiological, ecological and socio-behavioural factors that characterized MERS-CoV animalhuman interplay, valuable in assessing outbreak threat and severity and in guiding public health interventions cannot be overemphasized.

MERS-CoV, like $70 \%$ of all emerging infectious diseases, including Ebola, SARS, influenza, HIV/AIDS originated in wildlife, both bats and camels been postulated to be involved in MERS emergence $[1,8]$. Studies of camels in Saudi Arabia and Northern Africa indicated that the majority of cases have an history of direct or indirect contact with infected animal's secretions including milk, meat or other animal or contaminated products and humanto-human transmission in hospitals (nosocomial infections) and household(s) $[1,4,8,9]$. Cases of Human MERS-CoV infections have been reported in Northern Africa regions such as Tunisia, Algeria and Egypt has a history of direct or indirect contact with infected animal secretions including milk, meat or other animal or contaminated products and human-to-human transmission in hospital(s) and home(s) (nosocomial infections) $[1,4,5,7,9]$. The situation is complicated with disease outbreak illiteracy in most African communities coupled with infection route(s) and precise mechanism not yet well understood [1,8]. Moreover, unclear impact of varied levels of acquired immunity and those with compromised immune systems or elderly, weak quarantine and isolation facilities, poor individual innate system and lack of herd immunity or co-infections with other pathogens as well as MERS-CoV adaptations, environmental and climate changes can influence susceptible population and survivor's heterogeneity and variability $[9,10]$. Whereas most human cases have been reported mainly in Middle East, were sporadic and without sustained community transmission of imported cases into Europe, North America and Asia pacific fuelled concerns $[1,3,11]$ Yet, the MERS-CoV and other emerging infectious diseases threat and outbreak community that is expected to trigger health workers, community and public health emergency preparedness in emergency conditions are not clearly defined and potent public health threats call for improved disease prevention and control systems in Africa countries and elsewhere.

This paper discourses the importance of community alertness / vigilance and preparedness as well as robust surveillance and response capacity in averting MERS-CoV threat and looming epidemic. Significantly, also to galvanize and to foster leadership, policies, responsibility, increasing public health allocation and robust resilience from African governments and international community towards population health and wellness, upholding local public health emergency response plans and global health security.

Effective leadership and governance model, and coordination of sustainable strategies to governments and stakeholders (WHO, UN agencies, NGOs, humanitarian organizations and other actors) are of critical importance in financial resources mobilization and resources sharing in increasing evidence-based programs and interventions. Leveraging on public private partnerships (PPPs) are medium to long-term relationship between the public and private sector to deliver care assets and services underpinning public health services and community outcomes, and to ensuring the delivery of well-maintained, cost-effective public infrastructure and quality care services. The importance of diligent planning and priorities in supporting enablement and skill-building, stability and recovery are added value in emergency responses deliveries [11]. Such commitments and efforts have the goals and responsibilities to provide Africans and global community a comprehensive understanding of the MERSCoV animal reservoir-human interactions, disease transmission and etiology towards population capacity development, mass literacy and awareness campaigns, promoting trust and intensifying care support systems on MERS emerging threat and outbreak response, while integrating the whole routine best practices and adherence to safety standards and measures $[8,12]$ .Furthermore strengthening the local health systems leadership and coordination for planning, surveillance and early warning alert risk assessment, and enhanced vigilance through integrated public health emergencies MERS-CoV partnership approaches and programs in early risk communication, infection control, support upgrading of public health preparedness through improved national/regional laboratory diagnostics and surveillance systems $[8,9]$.

Community alertness, resilience, advocacy and mitigation is very vital in improving disease awareness, civil responsibility, and personal security on health risks prevention and protection against potential threat, fear, misinformation, perceptions, mistrust and resistance $[13,14]$. This provides preparedness opportunities and community resilience in realizing their full potential using multiple approaches and integrated program in multiple sectors such as education, arts and health education on standard of living, sanitation and hygiene (WASH) in urban and rural village dwellers, building trust and quality relationships and promoting collaborative advocacy, governance and discovery in improving lives and cooperative learning. In such facilitation 
Table 1: Vital approaches and activities to avert MERS-CoV and other emerging zoonotic diseases threat and epidemics.

\begin{tabular}{|c|c|}
\hline Approaches in emerging threat / epidemic & Response activities \\
\hline Effective leadership and governance & $\begin{array}{l}\text { Stakeholders commitments in leadership an governance } \\
\text { Timely financial resources mobilization in threat / crisis } \\
\text { Technical support assistance and policies } \\
\text { Diligent planning and coordination strategies } \\
\text { Resources and information sharing } \\
\text { Cooperation and partnership, and enabling environment }\end{array}$ \\
\hline Risk communications & $\begin{array}{c}\text { Evidence-based information communication } \\
\text { Simple, concise clear with compassion, } \\
\text { Focus and consistent, public trust and credible, } \\
\text { Comprehensive and shared freely to facilitate compliance } \\
\text { Provide preventive guidelines and safety measures for informed } \\
\text { decisions and behavioral changes }\end{array}$ \\
\hline Community Alertness, Advocacy and Mitigation & $\begin{array}{l}\text { Social mobilization and community engagement } \\
\text { Awareness campaign and outreach } \\
\text { Health education and health promotion } \\
\text { Early notification and fostering health seeking and utilization } \\
\text { Strengthening resilience and WASH measures }\end{array}$ \\
\hline Surveillance and early warming alert systems & $\begin{array}{l}\text { Understanding the fundamental epidemiological, ecological and socio- } \\
\text { behavioural factors information and data gathering } \\
\text { Improved diagnostics and early detection assays } \\
\text { Timely humanitarian emergency response }\end{array}$ \\
\hline Preparedness and Readiness( community and health systems) & $\begin{array}{l}\text { Infrastructure and facilities building and upgrading } \\
\text { Innovative health systems resilience development } \\
\text { Improved national/regional laboratory diagnostics and surveillance } \\
\text { systems } \\
\text { Resources and capacity development } \\
\text { Stockpile and chain supply management systems } \\
\text { Training, workshops and community empowerment }\end{array}$ \\
\hline Health systems responses programs and activities implementation & $\begin{array}{l}\text { Intensive case investigation and prompt identification } \\
\text { Resources and capacity development } \\
\text { Prompt and effective treatment (management) of cases } \\
\text { Emergency humanitarian response units and activities } \\
\text { Strengthening infection-prevention measures } \\
\text { Control programs implementation } \\
\text { Health care provisions and service delivery } \\
\text { Recovery and rehabilitation facilities }\end{array}$ \\
\hline Surveillance, Monitoring and Evaluation systems adaptations, & $\begin{array}{l}\text { Improved quarantine and isolation preventive and control measures } \\
\text { Evidence-based point of care and field surveillance and risk mitigation } \\
\text { strategies } \\
\text { Integrated of inter-sectoral and trans-disciplinary cross country } \\
\text { approaches and health systems policies } \\
\text { Enhanced Tracing and Tracking systems } \\
\text { Innovative and transformative epidemics investment and interventions } \\
\text { at all levels }\end{array}$ \\
\hline Certification of epidemic free & $\begin{array}{l}\text { WHO visitation and assessment of certification criteria } \\
\text { Upholding local public health emergency response plans and global } \\
\text { health security } \\
\text { Quality healthcare and outcome coupled with economic prosperity }\end{array}$ \\
\hline
\end{tabular}

of critical thinking about the complex intersections defined and rooted principles is critical in strengthening infection-prevention measures and social justice [14]. There is a need to increase community awareness and health promotion about diseases outbreak, early notification and fostering health seeking and utilization accessible, and culturally-behavioral relevant changes in most remotes communities [15]. Engagement of traditional and community leaders can greatly increase the percentage of health centers and formal and non-formal education institutions or centers to revamp up hopes and hypes in supporting the ongoing Ebola, polio, measles, TB and meningitis immunization campaigns, reducing poverty diseases vicious cycle, increase family earnings, productivity and sustainable development [15].

Operational viral diagnostic, surveillance and monitoring response indicators approaches and tools in MERS-CoV epidemic threat and crisis can be achieved by improving existing prognostic factors, laboratory facilities in both urban and rural areas in Africa. These include serological testing such as enzyme-linked immuno-sorbent assay (ELISA), immunofluorescence assay (IFA) to provide a robust for evidence of active infection and characterization of body immune system exposure to virus and 
presence of antibodies to MERS-CoV. This is very critical for active case finding, contact tracing and monitoring; quarantine of asymptomatic high risk contacts at home or in hospital quarantine facilities and isolation as well as prompt and effective treatment of laboratories confirmed cases [15,16]. Applications of molecular assays such as the real-PCR and post-sequencing genomics analysis for rapid detection of viral RNA in hosts are increasing role of disease outbreak and disaster surveillance, point of care and field diagnostics and surveillance [17] Strengthening robust and integrated health systems to support clinical (point of care) settings are fundamental for investigation purposes that are less labor-intensive rapid, available, accessible and cost effective to most vulnerable populations $[18,19]$ Operative infectionprevention and control measures rely on the effectiveness of preparedness, regional alert and sensitive early warning alert and continuous surveillance indicators approaches and tools and national guidelines against MERS are essential.

Effective and coordinated logistics in risk communication, planning and response is imperative in outbreak threat and epidemic management. Evidence-based information communication should be simple, clear with compassion, concise but focused and consistent, credible to gain public trust, comprehensive and shared freely. This is to facilitate compliance with preventive guidelines and safety measures or informed decisions, behavioural changes and enhanced surveillance and tracing strategies [20, 21]. However, communications landscape is evolving rapidly, and engaging the public health and safety personnel, especially during the times of crisis can be staggering. Socio media can facilitate access to and democratization of information, emergency risk communication, and interactive communication making use of public input, content exchange and partnerships, strategic public engagement and awareness, innovative strategies in public health emergencies. Despite this could improve population coordinated response, it presents some important pitfalls such as lack of confidentiality and non-verified or crude information [22]. The need to develop and implement programme to prevent MERS-CoV outbreak resurgence and control future transmission dynamics can be achieved by keeping animal-human record and track (archive) of the local or trans-boundary migration and travel history in parks or dromedary tours farms and stations as well as consumption of bat-dromedary related products. Effective implementation of appropriate risk communication measures is essential in prevention and management by understanding public perspective and risk/threat as a major tool in public health preparedness and response (e.g.: building trust, early announcement and information, transparency, preventive measures) and coordinated planning alliances, empathy, respect and actions based on communication surveillance in One Health Surveillance Network (OHSN).

MERS-CoV in vivo and clinical activities including relevant doses activity in pre-clinical studies on animal models, performance of feasibility of intra- or inter-countries surveys are essential to identify suitable hotspots and capacities for clinical research studies [23,24], building mass screening (e.g.: Hajj pilgrims), quarantine and isolation facilities and guidelines including blood transfusion donors both to health care providers or health workers and patients $[25,26,27]$. This is important in strengthening personal protective measures supported with medical services, surgical masks, evacuation facilities, rigorous hygiene practices such as hand-washing with soap and warm water, hospitalhygiene practices and personal hygiene should be of concern in the healthcare settings $[8,22,24]$. The need to develop a multicenter case-control series studies to characterize emerging diseases, infection and pathogenesis course, transmission and mechanism is also beneficial to clinical trials and the development of more sensitive and rapid diagnostics and integrating diagnostic information into decision making, safe and effective antiretroviral drugs and vaccines $[25,26]$.

Development and implementation of community-based national epidemic and disaster actionable insurance and compensation scheme that defines diseases epidemic or disaster insurance policies and emergency facility by risk insurers can be of tremendous benefits to expand national health insurance coverage and utilization in prevention, management of threat or crisis. It can be critical in provision of social, psychological and economic recovery and rehabilitation facilities or services across individual, family and community lifespan. There is a need to establish "safe epidemic coverage shelter" or "affordable catastrophic" insurance to facilitate that those traumatized or affected populations have the chance to benefit from timely and proven effective and efficient socio-psychological and healthcare services (e.g.: counselling, awareness and education campaigns, nursing care) pre-, during and post- public health threats or crises, and economic restorations on the face stigmatization, dignity impounded, psychosocial trauma and related health consequences mainly to vulnerable groups and survivors. In addition proper guidance and counselling to individual, family and community can be promoted by innovative transformation methods, cultural therapy, trainings and participation as well as legal support. Much needed partnership programmes and activities can be established in improving alertness and sustaining quality health in averting emerging outbreak threat and crisis in African inhabitants and elsewhere.

\section{Forward priorities to health ministries and govern-}

\section{ments in Africa}

The role of African Union (UA) and all stakeholders in providing committed leadership, governance and investment on operational and translational research initiatives to address MERS-CoV and other emerging infectious diseases research gaps is paramount towards rapid development of novel, safe and effective MERS-CoV chemotherapeutic agents and vaccines.

$>$ The need to establish local and sub-regional longitudinal studies in understanding the spatio-temporal patterns of MERS-CoV and of animal-human reservoir and humanhuman infectivity and transmissibility persistence.

$>$ Building of screening and surveillance laboratories facilities in improving early warning data and quantitative surveillance indicators for better understanding of risk factors mitigation, inform and guide humanitarian emergency response. 
Improve safe isolation and quarantine facilities capacity (human resources capacity development) as well as enhanced all-inclusive prevention, control and containment measures.

$>$ Harnessing the importance of digital publichealth technology applications, social media and web-based communication in mapping the risk factors and determinants and tracking geographical MERS-CoV distribution and hotspots for evidence-based implementation of emergency response plans nationwide and regionally.

$>$ Analyze the effectiveness of existing diagnostic and surveillance tools development to support animal-human investigations in animal-human risk exposure to milk, meat and urine contaminated products and traditional practices in different age groups in farms, camels and related animals including pets, shepherds, slaughter and meat vendors facilities, pets and livestock markets, tourism and racing locations.

$>$ Exploring innovative monitoring and evaluation infectious diseases approaches the environmental, farming, climate changes; vectors and pesticide control, pollution and waste management on MERS-Co emergence and health effects towards better environmental, climatic and veterinary public health services in building local to regional strategic preparedness and stockpile system and access to healthcare.

$>$ Nurturing integrated evidence-based nationwide surveillance and response capacity and targeted strategies to deal with emerging outbreaks and understanding human-animal interface in increasing urbanization, globalization of trade and travel in upholding "One Health" and global health security.
- Consolidating knowledge gains and best practices with cutting-edge integrated inter-sectoral and transdisciplinary partnership veterinary, agriculture and health, coupled with community empowerment and resilience is vital and of crucial impact in nurturing local, national and global health partnership, cooperation and coordination.

\section{Conclusions}

The urgent need of governments and global community to galvanize robust political leadership and governance commitment and financial investment to scale up the development and implementation of emerging outbreak alertness, preparedness and mitigation strategies require immediate attention through public health service administrators, policy-makers, legislators, heads of missions, academics and other professionals at all levels. The urgent need for effective rapid diagnostic kits for animalpopulation based screening and early detection, surveillance and monitoring systems in improving public health preparedness reinforce resilience in infection control measures and best practices in communities and healthcare settings. Furthermore strengthening the sensitivity of field and laboratory diagnostic screening, detection and testing capacity, timely access to highly effective quality assured diagnostic kits and rapid turnaround of results for prompt treatment. Importantly, intensification of social mobilization and mass literacy awareness, effective risk monitoring and communication in MERS-CoV susceptible countries are critical in improving public health services delivery, environmental and global health surveillance and response systems, evidence-based policies, priorities in diseases prevention and control programs.

\section{Acknowledgments}

No funding support was received on the project. 


\section{References}

1 WHO (2015) WHO guidelines for investigation of cases of human infection with Middle East Respiratory Syndrome Coronavirus (MERS-COV).

2 Gardner LM, MacIntyre CR (2014) Unanswered questions about the Middle East respiratory syndrome coronavirus (MERS-CoV). BMC Res Notes 7: 358.

3 Su S, Wong G, Liu Y, Gao GF, Li S,et al. (2015) MERS in South Korea and China: A Potential Outbreak Threat? Lancet 385: 2349,2350.

4 Oboho IK, Tomczyk SM, Al-Asmari AM, Banjar AA, Al-Mugti H, et al. (2014) MERS-CoV outbreak in Jeddah--a link to healthcare facilities. N Engl J Med 372: 846-854.

5 Chantal Reusken (2014) Geographic distribution of MERS-CoV among dromedary camels, Africa. Emerg Inf Diseases 20.

6 Bhutta ZA, Sommerfeld J, Lassi ZS, Salam RA, Das JK (2014) Global burden, distribution, and interventions for infectious diseases of poverty. Infect Dis Poverty 3: 21.

7 Kupfer SK (2015) Infectious diseases MERS surges again, but pandemic jitters ease. Science 347: 1296,1297.

8 Tambo E, Ugwu EC, Ngogang JY (2014) Need of surveillance response systems to combat Ebola outbreaks and other emerging infectious diseases in African countries. Infect Dis Poverty 3: 29.

9 Zhou XN, Bergquist R, Tanner M (2013) Elimination of tropical disease through surveillance and response. Infect Dis Poverty 2:1.

10 Woolhouse ME, Dye C, Etard JF, Smith T, Charlwood JD, et al.(1997) Heterogeneities in the transmission of infectious agents: Implications for the design of control programs. Proc Natl Acad Sci 94: 338-342.

11 Johns MC, Burke RL, Vest KG, Fukuda M, Pavlin JA, et al.(2011) A growing global network's role in outbreak response. BMC Public Health 11: S3.

12 Ge Q, Yao Z, Wang T, Liu Z, Li A, et al.(2014) Risk factors of the occurence and death of acute respiratory distress syndrome: $A$ prospective multicenter cohort study. Zhonghua Wei Zhong Bing Ji Jiu Yi Xue 26: 773-9.

13 13. Penttinen PM, Kaasik-Aaslav K, Friaux A, Donachie A, Sudre $B$, et al.(2013) Taking stock of the first 133 MERS coronavirus cases globally-ls the epidemic changing? Euro Surveill 18: 20596.

14 Halloran ME, Longini IM (2014) Emerging, evolving, and established infectious diseases and interventions. Science 345: 1292-1294.
15 Memish ZA, Cotten M, Watson SJ, Kellam P, Zumla A, et al. (2014) Community case clusters of Middle East respiratory syndrome coronavirus in Hafr Al-Batin, Kingdom of Saudi Arabia: A descriptive genomic study. Int J Infect Dis 23: 63-68.

16 Spanakis N, Tsiodras S, Haagmans BL, Raj VS, Pontikis K, et al. (2014) Virological and serological analysis of a recent Middle East respiratory syndrome coronavirus infection case on a triple combination antiviral regimen. Int J Antimicrob Agents 44: 528-532.

17 Lu X, Whitaker B, Sakthivel SK, Kamili S, Rose LE, et al. (2014) Real-time reverse transcription-PCR assay panel for Middle East respiratory syndrome coronavirus. J Clin Microbiol 52 : 67-75.

18 de Sousa R, Reusken C, Koopmans M (2013) MERS coronavirus: Data gaps for laboratory preparedness. J Clin Virol 59 : 4-11.

19 Waidyanatha N, Dubrawski A, M. G, Gow G (2011) Affordable System for Rapid Detection and Mitigation of Emerging Diseases. Intl Jof E-Health and Medical Communications 2: 73-90.

20 World Health Organization (2015) Middle East respiratory syndrome coronavirus (MERS-CoV): Summary of Current Situation, Literature Update and Risk Assessment.

21 European Center for Disease Control and Prevention (2014) Severe respiratory disease associated with Middle East respiratory syndrome coronavirus (MERS-CoV). Updated risk assessment.

22 22. Fung $I C H$, Fu KW, Ying $Y$, Schaible B, Hao Y, et al.(2013) Chinese social media reaction to the MERS-CoV and avian influenza $A(H 7 N 9)$ outbreaks. Infect Dis of Poverty 2: 31.

23 23. Saad M, Omrani AS, Baig K, Bahloul A, Elzein F, et al. (2014) Clinical aspects and outcomes of 70 patients with Middle East respiratory syndrome coronavirus infection: A single-center experience in Saudi Arabia. Int J Infect Dis. 29: 301-306.

24 24. Xia S, Liu Q, Wang Q, Sun Z, Su S, et al.(2014) Middle East respiratory syndrome coronavirus (MERS-CoV) entry inhibitors targeting spike protein. Virus Res 194: 200-210.

25 25. Hotez PJ, Bottazzi ME, Tseng CT, Zhan B, Lustigman S, et al.(2014) Calling for rapid development of a safe and effective MERS vaccine. Microbes Infect 16: 529-531.

26 26. Spengler JR, Chakrabarti AK, JoAnn D, et al. (2015) Utility of Oral Swab Sampling for Ebola Virus Detection in Guinea Pig Model. Emerg Infect Dis 21: 10 .

27 27. Watson JT, Hall AJ, Erdman DD, Swerdlow DL, Gerber SI (2014) Unraveling the Mysteries of Middle East Respiratory Syndrome Coronavirus. Emerg Infect Dis 20: 6. 